\title{
Pharmacogenetics of hypersensitivity drug reactions
}

\author{
Simone Negrini ${ }^{a, *}$, Laurent Becquemont ${ }^{\mathrm{b}}$
}

\begin{abstract}
a Department of internal medicine, clinical immunology unit, centre of excellence for biomedical research, university of Genoa, 16132 Genoa, Italy

b Pharmacology department, faculty of medicine Paris-Sud, UMR 1184, CEA, DSV/IMETI, division of immuno-virology, IDMIT, Inserm center for immunology of viral infections and autoimmune diseases, hôpital Bicêtre, university Paris Sud, Assistance publique-Hôpitaux de Paris, 94275 Le Kremlin-Bicêtre, France
\end{abstract}

Received 5 July 2016; accepted 2 September 2016

Available online 3 January 2017

\section{KEYWORDS \\ Hypersensitivity drug reactions; \\ HLA; \\ Abacavir; \\ Carbamazepine; \\ Allopurinol}

\begin{abstract}
Summary Adverse drug reactions are a significant cause of morbidity and mortality and represent a major burden on the healthcare system. Some of those reactions are immunologically mediated (hypersensitivity reactions) and can be clinically subdivided into two categories: immediate reactions (IgE-related) and delayed reactions (T-cell-mediated). Delayed hypersensitivity reactions include both systemic syndromes and organ-specific toxicities and can be triggered by a wide range of chemically diverse drugs. Recent studies have demonstrated a strong genetic association between human leukocyte antigen alleles and susceptibility to delayed drug hypersensitivity. Most notable examples include human leukocyte antigen (HLA)B*57:01 allele and abacavir hypersensitivity syndrome or HLA-B*15:02 and HLA-B*58:01 alleles related to severe cutaneous reactions induced by carbamazepine and allopurinol, respectively. This review aims to explore our current understanding in the field of pharmacogenomics of HLA-associated drug hypersensitivities and its translation into clinical practice for predicting adverse drug reactions.
\end{abstract}

(C) 2017 Société française de pharmacologie et de thérapeutique. Published by Elsevier Masson SAS. All rights reserved.

\section{Abbreviations}

ADRs adverse drug reactions APC antigen-presenting cell DIHS drug-induced hypersensitivity syndrome

* Corresponding author. Department of internal medicine, university of Genoa, viale Benedetto XV, 6, 16132 Genoa, Italy.

E-mail address: negrini@unige.it (S. Negrini).

$\begin{array}{ll}\text { EMA } & \text { European Medicines Agency } \\ \text { FDA } & \text { Food and Drug Administration } \\ \text { HDRs } & \text { hypersensitivity drug reactions } \\ \text { HIV } & \text { human immunodeficiency virus } \\ \text { HLA } & \text { human leukocyte antigen } \\ \text { MPE } & \text { maculopapular eruption } \\ \text { NPV } & \text { negative predictive value } \\ \text { PPV } & \text { positive predictive value } \\ \text { SJS } & \text { Stevens-Johnson syndrome } \\ \text { TCR } & \text { T-cell receptor }\end{array}$

EMA European Medicines Agency

FDA Food and Drug Administration

HDRs hypersensitivity drug reactions

HIV human immunodeficiency virus

MPE maculopapular eruption

NPV negative predictive value

PPV positive predictive value

TCR $\quad$ T-cell receptor 
TEN

toxic epidermal necrolysis

WHO World Health Organization

\section{Hypersensitivity drug reactions}

Adverse drug reactions (ADRs) are defined by the World Health Organization (WHO) as an "unintended, noxious response to a drug that occurs at a dose usually prescribed for human patients" [1]. ADRs are recognized as a major health problem, in the United States they are responsible of $6-7 \%$ of hospitalization cases and represent the fourth to sixth leading cause of death accounting for 100,000 fatal cases annually [2]. In Europe, it is estimated that $5 \%$ of all hospital admission and 197,000 deaths per year are caused by ADRs [3]. Along with the mortality and morbidity burden, it is estimated that the total cost to society of ADRs is $\$ 177$ billion and $€ 79$ billion in the USA and Europe, respectively $[3,4]$. Moreover, ADRs remain a huge cost burden for pharmaceutical industry representing the main cause of drug withdrawal from the market [5].

The historical pharmacologic classification of ADRs by Rawlins and Thompson classifies these into two main categories [6]. Type A reactions are predictable, dosedependent, and related to pharmacologic properties of the drug, and Type $B$ reactions, accounting for approximately $10 \%$ of all ADRs, are unpredictable, not dose-dependent and correspond to hypersensitivity drug reactions (HDRs). Occurring only in susceptible individuals Type B ADRs are sometimes termed "idiosyncratic". Since, terminology used to define HDRs is often confusing due to a lack of commonly accepted definitions, the European Academy of Allergology and Clinical Immunology published a statement paper in order to standardize the nomenclature of allergic diseases, also including drug allergy [7]. The revised nomenclature has been further adopted by the World Allergy Organization [8]. The proposed classification define as "drug allergy" those HDRs in which immunologic mechanisms may be demonstrated, while HDRs with symptoms and signs similar to real allergies but not triggered by a specific immunological mechanism are referred to as "non-allergic drug hypersensitivity" (e.g. non-specific histamine release, arachidonic acid pathway activation, bradykinin pathway alteration, etc.). With regard to the onset of symptoms, drug allergies may be further classified as immediate or delayed, suggesting also the immunological mechanism underlying the reaction $[7,8]$.

\section{Immunological basis of hypersensitivity drug reactions}

Immediate reactions occur immediately after drug exposure, are lgE-dependent, and derive from mast cell degranulation and release of pro-inflammatory mediators. Clinical manifestations include erythema, urticaria, angioedema, bronchospasm, and anaphylactic shock. Delayed hypersensitivity reactions generally occur days, or even weeks, after drug exposure and are mediated by antigen-specific $T$ lymphocytes. Examples of delayed-type HDRs include both systemic syndromes (e.g. drug rash with eosinophilia and systemic symptoms) and organ-specific toxicities (e.g. hepatitis, pneumonitis, etc.). Delayed-type hypersensitivity reactions correspond to type IV hypersensitivity according to the classification system of Gell and Coombs [9]. Since T-cells can orchestrate different immune responses resulting in different clinical entities, delayed reactions can be further classified into IVa (Th1 cells), IVb (Th2 cells), IVc (cytotoxic T-cell), and IVd (neutrophils) [10].

Since drugs are generally too small to stimulate an immune response (molecular weights $<1000$ Daltons), several, non-mutually exclusive, models have been proposed to explain how drugs can become immunogenic and trigger T-cells: the "hapten/pro-hapten model", the "Pi (pharmacologic interaction with immune receptors) concept", the "altered peptide repertoire hypothesis" and the "danger signal" theory. The "hapten/pro-hapten model" proposes that a chemically reactive drug, acting as hapten, can bind covalently to self-proteins (carriers) creating fully antigenic complexes. These neo-antigens are processed by an antigen-presenting cell (APC), loaded onto the HLA molecules and then presented to appropriate T-cells. The pro-hapten is a chemically non-reactive drug that becomes reactive upon metabolism [11]. According to "pi-concept hypothesis", a chemically inert drug, unable to covalently bind to proteins, is able to structurally "fit" into the Tcell receptor. This interaction needs neither metabolism nor antigen processing. The initial stimulation of the Tcell receptor (TCR) is further supplemented by TCR-HLA interaction and probably must take place in a context of hyper-reactive T-cells with a low threshold level of activation [12-14]. The "altered peptide repertoire hypothesis" proposes that the drug binds the antigen-binding groove of HLA thus modifying the antigen-binding cleft thus altering the repertoire of self-peptides that are bound and presented. Since T-cells are educated to be tolerant to a specific pool of peptides during thymic maturation, the presentation of these neo-self-peptides may induce T-cell activation. Recent observations provide strong evidence that this model is implicated in HDRs related to abacavir and carbamazepine [15]. Last hypothesis proposes that the drug itself or concomitant situations (e.g. viral infections) can provide "danger signals" capable of upregulating costimulatory molecules and cytokines in innate immune cells, thus facilitating the immune activation [11].

The human leukocyte antigen (HLA) molecules plays a crucial role in T-cells activation by presenting processed antigens to the T-cell receptor expressed on $\mathrm{T}$ lymphocytes. Broadly speaking, there are two main types of HLA molecules: the HLA class I molecules, expressed on most nucleated cells, and the HLA class II molecules, expressed by APCs, such as monocytes or dendritic cells. HLA class I molecules are encoded by three loci known as HLA-A, HLA$B$, and HLA-C; HLA class II molecules are encoded by three loci known as HLA-DR, HLA-DQ, and HLA-DP. HLA class I and class II molecules initiate the adaptive immune response by presenting antigens to CD8+ (cytotoxic) and CD4+ (helper) T-cells. Because the HLA molecules need to present an huge variety of "self" and "non-self" peptides, the HLA genes are both numerous and extremely polymorphic. Taking into account the crucial role of HLA in immune response it is not surprising that particular HLA alleles have been associated with susceptibility to diseases in which the immune system is considered the principal mediator, like infectious or autoimmune disorders $[16,17]$. In the same way, certain HLA alleles 
have been associated with an increased risk of delayed HDRs. The evidence that certain HLA alleles may increase the risk of HDRs has prompted the development of screening test strategies and labeling changes to drug information sheets.

To date, the best-characterized HLA-HDRs associations include abacavir, carbamazepine, and allopurinol.

\section{Specific examples of HLA-related hypersensitivity drug reactions}

\section{Abacavir}

Abacavir is a nucleoside analog reverse-transcriptase inhibitor used as part of combination therapy for the treatment of human immunodeficiency virus (HIV) infection. Abacavir is generally well tolerated, but $5-8 \%$ of patients may develop an hypersensitivity reaction generally within the first 6 weeks of treatment [18]. Abacavir hypersensitivity reaction is clinically characterized by fever, rash, constitutional, gastrointestinal and respiratory symptoms. Symptoms worsen with the continuation of abacavir and can be life-threatening if the drug is re-administered after discontinuation [19]. Even if Abacavir hypersensitivity was reported in the pre-marketing phase of its development, only in 2002, two independent groups described a strong association between the HLA-B*57:01 allele and susceptibility to develop abacavir hypersensitivity syndrome [20,21].

This association was successively reported by different groups [22-25], but it was definitively confirmed in 2008 with the results of the largest randomized clinical trial performed to date in pharmacogenetics: the PREDICT-1 trial. In this study, 1956 patients from 19 countries were randomized to either HLA-B*5701 genetic screening (carriers of HLA-B*5701 did not receive abacavir) or to the standard of care (no screening and all patients received abacavir). Genetic screening virtually eliminated all immunologically confirmed HDRs ( $0 \%$ in the screened group versus $2.7 \%$ in the control group), thus supporting a great translational potential of HLA-B*57:01 as screening test for abacavirrelated HDR [26]. Nevertheless, the generalisability of the PREDICT-1 results to all races was limited by its prevalent Caucasian population ( $84 \%$ of the enrolled patients were Caucasian). The SHAPE trial, a case-control study including similar percentages of black and white patients, revealed a $100 \%$ negative predictive value (NPV) of HLA-B*57:01 testing for abacavir hypersensitivity both for black and white patients, thus confirming the value of the screening across different ethnicities [27]. A recent meta-analysis further confirmed that HLA-B*57:01 carriage is significantly associated with abacavir-induced hypersensitivity reactions in Whites, Blacks, and Hispanics [28]. The NPV of HLA-B*57:01 screening is $100 \%$ (which means that HLA-B*57:01 negative individuals will not develop hypersensitivity) making this test highly valuable in predicting the risk of abacavir-related HDR. Nevertheless, the positive predictive value (PPV) of HLA- $B * 57: 01$ genetic testing is estimated to be around $50 \%$ implying that around half of all patients found positive at screening actually will develop the HDR if exposed to abacavir. High NPV and low PPV seems to be a characteristic feature of HLA screening tests (Table 1) [29-33] suggesting that these markers are necessary but not sufficient for ADR onset, and other genes and/or environmental factors should be involved.

Interestingly, HLA-B*57:01 has also been associated to an increased risk of drug-induced liver injury in patients treated with the antibiotic flucloxacillin [34]. The association of HLA-B*57:01 with both abacavir hypersensitivity and flucloxacillin-induced hepatotoxicity indicates that the same HLA allele lead to completely different reactions triggered by chemically unrelated drugs. In addition, HLA$B * 57: 01$ has been associated with slow or non-progression of HIV infection [35-37]. Taken together, these data seem to suggest that HLA-B*57:01 is related with an "hyperactive" immune response that, on one hand may increase the risk to develop hypersensitivity reactions, but, on the other hand, confer resistance to infections.

In light of the large number of evidences correlating HLA-B*57:01 and abacavir-induced HDR, the genetic screening prior to abacavir treatment is recommended by Food and Drug Administration (FDA), European Medicines Agency (EMA), and virtually all international HIV treatment guidelines [32,33]. Currently, HLA-B*57:01 is one of most, if not the most, commonly used pharmacogenetic marker in clinical practice.

\section{Carbamazepine}

Stevens-Johnson syndrome (SJS) and toxic epidermal necrolysis (TEN, also known as Lyell's syndrome) are severe mucocutaneous reactions characterized by extensive detachment of the skin. These skin reactions are considered to be variants of the same disease and they are classified according to the degree of skin involvement which is $<10 \%$ in SJS, and $>30 \%$ in TEN. A skin detachment of 10 to $30 \%$ of body surface area is named SJS/TEN overlap [38]. SJS/TEN are rare diseases with an incidence of 2-6 cases/million per year but both are associated with high morbidity and mortality (5\% and $50 \%$ mortality for SJS for TEN, respectively) $[38,39]$. From a pathophysiological points of view, SJS/TEN are attributable to autoreactive CD8+ cytotoxic lymphocytes that cause keratinocytes death through the release of cytotoxic molecules, such as soluble-FasL, granzyme B, perforin, granulysin and pro-apoptotic cytokines [40]. In the vast majority of cases SJS/TEN are triggered by drugs and several medications are considered at high risk of inducing SJS/TEN [41].

Carbamazepine is an aromatic amine anticonvulsant widely employed to treat epilepsy, bipolar disorder, and trigeminal neuralgia. Carbamazepine is generally safe, but has been associated with maculopapular eruption (MPE), drug rash with eosinophilia and systemic symptoms (DRESS) syndrome (also known as DIHS [drug-induced hypersensitivity syndrome]), and more rarely SJS/TEN [42]. In 2004, Chung et al. reported a very strong association between carbamazepine-induced SJS/TEN and the HLA-B*15:02 allele in Han Chinese patients from Taiwan. They observed that all patients with carbamazepine-induced SJS/TEN carried the HLA-B*15:02 allele as compared to only $3 \%$ of drugtolerant subjects and $8.6 \%$ of the general population [43]. Further studies have replicated this association in different Asian areas including China, Thailand, Malaysia, India, Vietnam, and Cambodia [44-53], and three recent meta- 


\begin{tabular}{|c|c|c|c|c|c|c|}
\hline Drug & $\begin{array}{l}\text { Level of } \\
\text { evidence } \\
\text { [29] }\end{array}$ & Allele & Clinical manifestation & Population & Risk of ADR & $\begin{array}{l}\text { Clinical implementation }[29,32,33] \\
\text { French National Pharmacogenetics } \\
\text { network (RNPGx) recommendations }\end{array}$ \\
\hline Abacavir & $1 \mathrm{~A}$ & HLA-B*57:01 & DRESS & Mixed population & Increased & $\begin{array}{l}\text { FDA: boxed warning, test } \\
\text { recommended, EMA: test required, } \\
\text { PMDA: label information, HCSC: } \\
\text { test recommended, CPIC: } \\
\text { alternative drug for positive } \\
\text { patients, test recommended } \\
\text { DPWG: alternative drug for positive } \\
\text { patients, virtually all HIV } \\
\text { Treatment Guidelines: test } \\
\text { required/recommended } \\
\text { PPV = 55\%, NPV = } 100 \% \text { for patch } \\
\text { test confirmed, NNT to prevent } \\
\text { "1"' = 32 } \\
\text { RNPGx reco: essential }\end{array}$ \\
\hline Acetazolamide & 4 & HLA-B*59:01 & SJS/TEN & Asian (Korean) & Increased & $\begin{array}{l}\text { RNPGx recommendation: no } \\
\text { indication in routine care }\end{array}$ \\
\hline Acetaminophen & 3 & HLA-DQB1*02:02 & SJS/TEN & Caucasian (Italian) & Increased & $\begin{array}{l}\text { RNPGx reco: no indication in } \\
\text { routine care }\end{array}$ \\
\hline \multirow[t]{10}{*}{ Allopurinol } & $1 \mathrm{~A}$ & HLA-B*58:01 & SJS/TEN & Mixed Population & Increased & European national competent \\
\hline & $2 B$ & HLA-A*33:03 & $\begin{array}{l}\text { Drug Hypersensitivity, } \\
\text { SJS/TEN }\end{array}$ & Mixed population & Increased & $\begin{array}{l}\text { authorities: warning (no genotyping } \\
\text { recommendations), ACR: }\end{array}$ \\
\hline & 3 & HLA-A*02:01 & SJS/TEN & Asian & Decreased & alternative drug for HLA-B*58:01 \\
\hline & 3 & HLA-B*48:01 & Drug Hypersensitivity & Asian & $\begin{array}{l}\text { Increased (not } \\
\text { in all studies) }\end{array}$ & $\begin{array}{l}\text { positive patients, CPIC: alternative } \\
\text { drug for HLA-B*58:01 positive }\end{array}$ \\
\hline & 3 & HLA-C*03:02 & Rash & Asian (Korean) & Increased & patients \\
\hline & 3 & HLA-C*08:01 & Drug Hypersensitivity & Mixed population & $\begin{array}{l}\text { Increased (not } \\
\text { in all studies) }\end{array}$ & $\begin{array}{l}\text { HLA-B*58:01 test } \\
\text { PPV }=3 \%, N P V=100 \% \text { in } \mathrm{Han}\end{array}$ \\
\hline & 3 & HLA-DQB1*05:02 & SJS/TEN & Caucasian (Italian) & Increased & Chinese, NNT to prevent " 1 "' $=250$ \\
\hline & 3 & HLA-DR9; HLA-DR14 & MPE & Asian & Increased & RNPGx reco: HLA-B*58:01 \\
\hline & 3 & $\begin{array}{l}\text { HLA-DRB1*03:01 (in LD } \\
\text { with HLA-B*58:01 in } \\
\text { some populations) }\end{array}$ & SJS/TEN & Asian (Han Chinese) & Increased & $\begin{array}{l}\text { potentially useful namely in Asian } \\
\text { ancestry populations (Han Chinese, } \\
\text { Thaï and Taiwanese) }\end{array}$ \\
\hline & 3 & $\begin{array}{l}\text { HLA-DRB } 1 * 15: 02 \\
\text { HLA-DRB } 1 * 13: 02\end{array}$ & SJS/TEN & Caucasian (Italian) & Increased & \\
\hline \multirow[t]{2}{*}{ Aspirin } & $2 B$ & HLA-DPB1*03:01 & Asthma & Mixed population & Increased & N/A \\
\hline & 3 & HLA-DPB1*04:01 & Asthma & Mixed population & Decreased & $\begin{array}{l}\text { RNPGx reco: HLA-B*58:01: no } \\
\text { indication in routine care }\end{array}$ \\
\hline
\end{tabular}




\begin{tabular}{|c|c|c|c|c|c|c|}
\hline Drug & $\begin{array}{l}\text { Level of } \\
\text { evidence } \\
\text { [29] }\end{array}$ & Allele & Clinical manifestation & Population & Risk of ADR & $\begin{array}{l}\text { Clinical implementation }[29,32,33] \\
\text { French National Pharmacogenetics } \\
\text { network (RNPGx) recommendations }\end{array}$ \\
\hline $\begin{array}{l}\text { Azathioprine (or } \\
\text { mercaptopurine) }\end{array}$ & 3 & $\begin{array}{l}\text { HLA-DQA1*02:01, } \\
\text { HLA-DRB1*07:01 }\end{array}$ & Pancreatitis & European & Increased & $\begin{array}{l}\text { N/A } \\
\text { RNPGx reco: no indication in } \\
\text { routine care }\end{array}$ \\
\hline \multirow[t]{12}{*}{ Carbamazepine } & $1 \mathrm{~A}$ & HLA-B*15:02 & SJS/TEN & Mainly Asian ${ }^{a}$ & Increased & $\begin{array}{l}\text { HLA- } B^{*} 15: 02 \\
\text { FDA: Boxed Warning, test required } \\
\text { (patients with Asian ancestry), } \\
\text { European national competent } \\
\text { authorities: warning, test } \\
\text { recommended (patients with Asian } \\
\text { ancestry), PMDA: label information, } \\
\text { HCSC: test recommended, CPNDS: } \\
\text { drug contraindicated for positive }\end{array}$ \\
\hline & $2 \mathrm{~A}$ & HLA-B*15:11 & SJS/TEN & $\begin{array}{l}\text { Asian (Japanese, } \\
\text { Korean, Han Chinese) }\end{array}$ & Increased & $\begin{array}{l}\text { patients } \\
\text { Asian: } \mathrm{PPV}=1.8 \%, \mathrm{NPV}=100 \%, \mathrm{NNT}\end{array}$ \\
\hline & $2 B$ & HLA-A*31:01 & DRESS, MPE, SJS/TEN & Mixed population ${ }^{a}$ & Increased & to prevent " $1 "=461$ \\
\hline & $2 B$ & HLA-B*40:01 & SJS/TEN & $\begin{array}{l}\text { Asian (Han Chinese, } \\
\text { Taiwanese) }\end{array}$ & Decreased & $\begin{array}{l}\text { HLA-A*31:01 } \\
\text { FDA: warning (no genotyping }\end{array}$ \\
\hline & $2 B$ & $\begin{array}{l}\text { HLA-C*03:02 (in LD } \\
\text { with HLA-B*58:01 in } \\
\text { some populations) }\end{array}$ & SJS/TEN & Mixed population & Increased & $\begin{array}{l}\text { recommendations), European } \\
\text { national competent authorities: } \\
\text { warning (no genotyping }\end{array}$ \\
\hline & 3 & HLA-B*15:18 & SJS/TEN & Asian (Japanese) & Increased & recommendations), HCSC: test \\
\hline & 3 & HLA-B*58:01 & MEP & Asian (Han Chinese) & Decreased & recommended, CPNDS: drug \\
\hline & 3 & HLA-B*58:01 & SJS/TEN & Asian (Han Chinese) & $\begin{array}{l}\text { Increased } \\
\text { (decreased in } \\
\text { one study) }\end{array}$ & $\begin{array}{l}\text { contraindicated positive patients } \\
\text { Japanese: } P P V=12 \%, \text { NPV }=99 \% \text {, } \\
\text { NNT to prevent "'1"' }=67\end{array}$ \\
\hline & 3 & HLA-B*59:01 & SJS/TEN & Asian (Japanese) & Increased & Caucasian: PPV $=43 \%, \mathrm{NPV}=92 \%$ \\
\hline & 3 & HLA-B*13:01 & Drug Hypersensitivity & Caucasian, Asian & $\begin{array}{l}\text { Increased (not } \\
\text { in all studies) }\end{array}$ & $\begin{array}{l}\text { NNT to prevent " } 1 " \text { " }=47 \\
\text { RNPGx reco: HLA-B"15:02 advisable }\end{array}$ \\
\hline & 3 & HLA-B*46:01 & SJS/TEN & Asian & Decreased & in Asian ancestry populations (Han \\
\hline & 3 & HLA-DRB $1{ }^{*} 03: 01$ & MEP & Asian (Han Chinese) & Increased & Chinese and Taiwanese) \\
\hline Clozapine & 3 & HLA-DRB3*02:02 & Agranulocytosis & Caucasian & Increased & $\begin{array}{l}\text { N/A RNPGx reco: no indication in } \\
\text { routine care }\end{array}$ \\
\hline \multirow[t]{2}{*}{ Dapsone } & $2 \mathrm{~A}$ & HLA-B*13:01 & Drug hypersensitivity & Asian & Increased & $\begin{array}{l}\mathrm{PPV}=7.8 \%, \mathrm{NPV}=99.8 \% \\
\text { NNT to prevent "'1"'= } 84 \\
\text { RNPGx reco: no indication in } \\
\text { routine care }\end{array}$ \\
\hline & 3 & HLA-A*02:01 & Drug hypersensitivity & $\begin{array}{l}\text { Asian (Japanese, } \\
\text { Korean, Chinese) }\end{array}$ & $\begin{array}{l}\text { Increased } \\
\text { (decreased in } \\
\text { one study) }\end{array}$ & $\begin{array}{l}\text { N/A RNPGx reco: no indication in } \\
\text { routine care }\end{array}$ \\
\hline
\end{tabular}




\begin{tabular}{|c|c|c|c|c|c|c|}
\hline Drug & $\begin{array}{l}\text { Level of } \\
\text { evidence } \\
\text { [29] }\end{array}$ & Allele & Clinical manifestation & Population & Risk of ADR & $\begin{array}{l}\text { Clinical implementation }[29,32,33] \\
\text { French National Pharmacogenetics } \\
\text { network (RNPGx) recommendations }\end{array}$ \\
\hline Lamotrigine & $\begin{array}{l}3 \\
3\end{array}$ & $\begin{array}{l}\text { HLA-B*58:01 } \\
\text { HLA-B*15:02 }\end{array}$ & $\begin{array}{l}\text { SJS/TEN } \\
\text { SJS/TEN }\end{array}$ & $\begin{array}{l}\text { Mixed population } \\
\text { Asian }\end{array}$ & $\begin{array}{l}\text { Increased } \\
\text { Increased (only } \\
\text { found in } \\
\text { meta-analyses) }\end{array}$ & $\begin{array}{l}\text { N/A RNPGx reco: no indication in } \\
\text { routine care }\end{array}$ \\
\hline Lapatinib & $2 B$ & HLA-DQA1*02:01 & $\begin{array}{l}\text { Drug-induced liver } \\
\text { injury }\end{array}$ & Mixed population & Increased & $\begin{array}{l}\text { HLA-DQA1*02:01 or } \\
\text { HLA-DRB1*07:01 alleles } \\
\text { carrier = FDA: warning (no } \\
\text { genotyping recommendations), } \\
\text { EMA: warning (no genotyping } \\
\text { recommendations), HCSC: warning } \\
\text { (no genotyping recommendations) } \\
\text { RNPGx reco: potentially usefull }\end{array}$ \\
\hline \multirow[t]{3}{*}{ Nevirapine } & $2 \mathrm{~A}$ & HLA-B*35:01 & $\begin{array}{l}\text { Drug Hypersensitivity } \\
\text { (rash) }\end{array}$ & Mixed population & $\begin{array}{l}\text { Increased } \\
\text { (reported to be } \\
\text { depend on CD4 } \\
\text { T-cells count) } \\
\text { Increased }\end{array}$ & $\begin{array}{l}\text { N/A } \\
\text { RNPGx reco: no indication in } \\
\text { routine care }\end{array}$ \\
\hline & 3 & HLA-B*35:01 & $\begin{array}{l}\text { (hepatitis) } \\
\text { Drug hypersensitivity } \\
\text { (rash) }\end{array}$ & Asian (Thai) & $\begin{array}{l}\text { Increased } \\
\text { (reported to be } \\
\text { depend on CD4 } \\
\text { T-cells count) }\end{array}$ & \\
\hline & 3 & HLA-DQB1*05:01 & $\begin{array}{l}\text { Drug hypersensitivity } \\
\text { (various } \\
\text { manifestations) }\end{array}$ & $\begin{array}{l}\text { Black or African } \\
\text { American }\end{array}$ & Decreased & \\
\hline
\end{tabular}




\begin{tabular}{|c|c|c|c|c|c|c|}
\hline Drug & $\begin{array}{l}\text { Level of } \\
\text { evidence } \\
\text { [29] }\end{array}$ & Allele & Clinical manifestation & Population & Risk of ADR & $\begin{array}{l}\text { Clinical implementation }[29,32,33] \\
\text { French National Pharmacogenetics } \\
\text { network (RNPGx) recommendations }\end{array}$ \\
\hline $\begin{array}{l}\text { Nonsteroidal } \\
\text { anti-inflammatory } \\
\text { drugs }\end{array}$ & 3 & HLA-DR11 & $\begin{array}{l}\text { Anaphylactoid } \\
\text { reactions }\end{array}$ & Spain & Increased & $\begin{array}{l}\text { N/A } \\
\text { RNPGx reco: no indication in } \\
\text { routine care }\end{array}$ \\
\hline \multirow[t]{4}{*}{ Oxcarbazepine } & 3 & HLA-B*13:02 & MEP & Asian (Chinese) & Increased & $\mathrm{N} / \mathrm{A}$ \\
\hline & 3 & HLA-B*15:02 & MEP, SJS/TEN & Asian & Increased & RNPGx reco: no indication in \\
\hline & 3 & $\begin{array}{l}\text { HLA-B*15:19; } \\
\text { HLA-B*15:27; } \\
\text { HLA-B*27:09; } \\
\text { HLA-B*38:02; } \\
\text { HLA-B* } 48: 04\end{array}$ & MEP & Asian (Han Chinese) & Increased & routine care \\
\hline & 4 & $\begin{array}{l}\text { HLA- } \\
B * 15: 18: 01 / * 40: 01: 01 \\
\text { genotype }\end{array}$ & SJS/TEN & Mixed population & Increased & $\begin{array}{l}\mathrm{N} / \mathrm{A} \\
\text { RNPGx reco: no indication in } \\
\text { routine care }\end{array}$ \\
\hline \multirow[t]{2}{*}{$\begin{array}{l}\text { Pegylated interferon } \\
\text { and ribavirin }\end{array}$} & 3 & HLA-B*38:01 & $\begin{array}{l}\text { Non-responder to } \\
\text { therapy }\end{array}$ & Egyptian & N/A & $\begin{array}{l}\text { DPWG: information about lower } \\
\text { response in HLA-B* } 44 \text { negative } \\
\text { patients (no genotyping } \\
\text { recommendations) }\end{array}$ \\
\hline & 3 & HLA-B*44:02 & Sustained response & Spain & N/A & $\begin{array}{l}\text { RNPGx reco: no indication in } \\
\text { routine care }\end{array}$ \\
\hline Phenobarbital & 3 & HLA-B*51:01 & SJS/TEN & Asian & Increased & $\begin{array}{l}\text { N/A RNPGx reco: no indication in } \\
\text { routine care }\end{array}$ \\
\hline \multirow[t]{3}{*}{ Phenytoin } & $1 \mathrm{~A}$ & HLA-B*15:02 & SJS/TEN & Asian & Increased & $\begin{array}{l}\text { FDA: warning (no genotyping } \\
\text { recommendations), CPIC: drug } \\
\text { contraindicated for } B^{*} 15: 02 \\
\text { positive patients } \\
\text { HLA-B }{ }^{*} 15: 02 \text { test: } P P V=33 \% \text {, }\end{array}$ \\
\hline & 3 & HLA-B*13:01 & SJS/TEN & Asian & $\begin{array}{l}\text { Increased (not } \\
\text { increased in } \\
\text { one study) }\end{array}$ & $\begin{array}{l}\text { NPV }=100 \% \\
\text { RNPGx reco: HLA-B*15:02 advisable } \\
\text { in Asian ancestry populations (Han }\end{array}$ \\
\hline & 3 & HLA-B*56:02 & DRESS & Indigenous Australian & Increased & Chinese and Taiwanese) \\
\hline \multirow[t]{2}{*}{ Sulfasalazine } & 3 & $\begin{array}{l}\text { HLA-B*39:01; } \\
\text { HLA-B*13:01 }\end{array}$ & DRESS & Asian & Increased & $\begin{array}{l}\text { N/A RNPGx reco: no indication in } \\
\text { routine care }\end{array}$ \\
\hline & 4 & HLA-B*15:05 & DRESS & Asian & Increased & \\
\hline
\end{tabular}




\begin{tabular}{|c|c|c|c|c|c|c|}
\hline Drug & $\begin{array}{l}\text { Level of } \\
\text { evidence } \\
\text { [29] }\end{array}$ & Allele & Clinical manifestation & Population & Risk of ADR & $\begin{array}{l}\text { Clinical implementation }[29,32,33] \\
\text { French National Pharmacogenetics } \\
\text { network (RNPGx) recommendations }\end{array}$ \\
\hline $\begin{array}{l}\text { Thioamides } \\
\text { (carbimazole, } \\
\text { methimazole, } \\
\text { propylthiouracil) }\end{array}$ & $2 \mathrm{~A}$ & HLA-B*38:02:01 & Agranulocytosis & Mixed population & Increased & $\begin{array}{l}\text { N/A RNPGx reco: no indication in } \\
\text { routine care }\end{array}$ \\
\hline Ticlopidine & 3 & HLA-B*44:03 & $\begin{array}{l}\text { Hepatotoxicity and } \\
\text { cholestatic } \\
\text { hepatotoxicity }\end{array}$ & Asian & Increased & $\begin{array}{l}\text { N/A RNPGx reco: no indication in } \\
\text { routine care }\end{array}$ \\
\hline Trichloroethylene & 3 & HLA-B*13:01 & $\begin{array}{l}\text { Hypersensitivity } \\
\text { dermatitis }\end{array}$ & Asian (Chinese) & Increased & $\begin{array}{l}\text { N/A RNPGx reco: no indication in } \\
\text { routine care }\end{array}$ \\
\hline Ustekinumab & 3 & HLA-C*06:02 & $\begin{array}{l}\text { Better response } \\
\text { (psoriasis) }\end{array}$ & Caucasian & $\mathrm{N} / \mathrm{A}$ & $\begin{array}{l}\text { N/A RNPGx reco: no indication in } \\
\text { routine care }\end{array}$ \\
\hline Ximelagatran & N/A & $\begin{array}{l}\text { DRB1*07:01, } \\
\text { DQA1*02:01 }\end{array}$ & $\begin{array}{l}\text { Drug-induced liver } \\
\text { injury }\end{array}$ & European & Increased & $\begin{array}{l}\text { Withdrawn in } 2006 \text { following } \\
\text { reports of hepatotoxicity }\end{array}$ \\
\hline Zonisamide & 3 & HLA-A*02:07:01 & SJS/TEN & Asian (Japanese) & Increased & $\begin{array}{l}\text { N/A RNPGx reco: no indication in } \\
\text { routine care }\end{array}$ \\
\hline \multicolumn{7}{|c|}{$\begin{array}{l}\text { ACR: American College of Rheumatology; CPIC: Clinical Pharmacogenetics Implementation Consortium; CPNDS: Canadian Pharmacogenomics Network for Drug Safety; DPWG: Dutch } \\
\text { Pharmacogenetics Working Group; DRESS: drug reaction with eosinophilia and systemic symptoms; EMA: European Medicines Agency; FDA: US Food and Drug Administration; HCSC: Health } \\
\text { Canada (Santé Canada); HDRs: hypersensitivity drug reactions; MPE: maculopapular eruption; NPV: negative predictive value; OR: odds ratio; PMDA: Pharmaceuticals and Medical Devices } \\
\text { Agency - Japan; PPV: positive predictive value; SJS: Stevens-Johnson syndrome; TEN: toxic epidermal necrolysis. RNPGx recommendations are categorized into } 4 \text { categories: essential } \\
\text { advisable, potentially usefull and no indication in routine care. } \\
\text { a See text for further details. }\end{array}$} \\
\hline
\end{tabular}


analyses that combined data obtain from different Asian population all found odds ratios of approximately 80 for carbamazepine-induced SJS/TEN in patients carrying the HLA-B*15:02 allele [54-56]. A 2011 large prospective study, intended to evaluate the value of HLA-B*15:02 genotyping before carbamazepine treatment, demonstrated that SJS/TEN did not develop in any of the treated patients if the subjects were HLA-B*1502-negative. Considered an expected incidence of $0.23 \%$ based on historical data, the genetic screening successfully prevented 10 cases of HDR [57]. These results paved the way for the implementation of HLA-B*15:02 screening before carbamazepine therapy in Asian patients. Nevertheless, HLA-B*15:02 has not been found to be a risk factor in Caucasian or Japanese populations, probably because of the very low prevalence of this allele in these ethnic group (1\% or less). In European or Japanese recent data suggests that carbamazepine-related HDRs, including SJS/TEN, MPE, and DRESS, are associated with the presence of the HLA-A*31:01 allele [54,58-61]. Interestingly, in Han Chinese, HLA-A*31:01 allele has been associated with an increased risk of carbamazepine-induced MPE or DRESS, while HLA-B*15:02 is related only to SJS/TEN $[27,46,54]$. These observations suggest that genetic susceptibility to carbamazepine-related HDRs, at least in certain populations, seems to be phenotype-specific. Beyond HLA$B * 15: 02$ and HLA-A*31:01, other HLA alleles have been associated to an increased risk of carbamazepine-related HDRs in various populations (Table 1), nevertheless these studies are limited and need further confirmation. At this time, FDA recommends HLA-B*15:02 genetic testing prior to a carbamazepine based treatment in individuals of Asian ethnicity $[32,33]$. A warning has been added to the prescribing information by FDA indicating that the risks and benefits of using carbamazepine should be weighed in HLA-A*31:01 allele carriers [33]. Different reports put HLA-B*15:02 in relation also with SJS/TEN induced by aromatic antiepileptic drugs, other than carbamazepine, such as phenytoin, oxcarbazepine and lamotrigine $[7,46,54,62]$. These findings suggest that HLA-B*15:02 positive patients may show cross-reactivity to structurally related anticonvulsants and consequently special attention should be given when considering antiepileptic treatment for HLA-B*1502 allele carriers [63]. Currently, FDA advise to avoid phenytoin as alternative for carbamazepine in HLA-B*1502 positive patients, although it does not make specific recommendations for other structurally-related anticonvulsant [33].

\section{Allopurinol}

Allopurinol is a xanthine oxidase inhibitor used to treat disorders associated with hyperuricemia, such as chronic gout and tumor lysis syndrome. The most serious ADRs associated to allopurinol are HDRs, which include DRESS and SJS/TEN and occur in approximately $0.1-0.4 \%$ of treated patients [64]. Allopurinol is the most frequent cause of SJS/TEN in Europe, exceeding carbamazepine and phenytoin, while in southeast Asia, is the second most common SJS/TENcausative drug after carbamazepine $[45,65]$.

In 2005, a case-controlled study reported that a specific HLA allele, HLA-B*58:01, was present in $100 \%$ of patients with allopurinol-induced HDRs compared to $15 \%$ of allopurinol-tolerant patients and $20 \%$ of healthy con- trols [66]. Similar results have been replicated in other ethnic groups: strong association was reported in Han Chinese, Thais, Japanese and Koreans while weaker association was described in Europeans [67-72]. This differences probably reflect the different prevalence of HLA-B*58:01 which is higher in Asian populations as compared to Europeans and/or additional contributing factors not yet elucidated. However, a recent meta-analysis found a strong and significant association the HLA-B*58:01 allele and allopurinol-induced SJS/TEN (OR 96.60 and OR 79.28 in studies with matched-control or population-control, respectively) in both Asian and non-Asian populations [73]. In the light of the strong association of HLA-B*58:01 with hypersensitivity reactions to allopurinol across different populations, a genetic screening of patients prior to initiating allopurinol will be most likely adopted in future. To date, FDA and EMA have not implemented allopurinol labeling information for HLA-B*58:01 testing. The Clinical Pharmacogenetics Implementation Consortium guidelines recommend that allopurinol should not be prescribed to patients who are positive for the HLA-B*5801 allele but underline that negative testing does not exclude the possibility of developing SCARs, especially in European populations [64]. The 2012 revised guidelines of the American College of Rheumatology also recommend HLA-B*58:01 screening prior to allopurinol prescription, especially in those populations with high frequencies of the allele, such as the Han Chinese or Thais [74].

\section{Conclusion}

Abacavir, carbamazepine, and allopurinol are key examples of pharmacogenetics implementation in routine medical practice (Table 2) [75], but many other HLA-related HDRs have been reported so far (Table 1). However, several considerations should be taken into account before such associations could be translated in cost-effective screening procedures, these factors have been recently reviewed by Phillips and Mallal [76]. Premises for a successful implementation of a given HLA pharmacogenetic testing into routine clinical care requires that: drug toxicity is severe and persistent, the NPV (and ideally also the PPV) of the test reach $100 \%$, culprit HLA allele is prevalent inside the screened population, and the number of patients needed to test in order to prevent a case is low. In addition, responsible drug should have good efficacy, cost-effectiveness and, tolerability, in the absence of alternative drug(s) with similar positive characteristics. Flucloxacillin-related liver toxicity can be cited as a paradigmatic example, although strongly associated with HLA-B*57:01 allele (OR 80), almost 14,000 patients should be tested to prevent a single case of hepatotoxicity, making this screening neither cost-effective nor feasible $[34,77]$. In view of this, only few HLA-disease-drug associations reported to date will be reasonably translated into routine clinical practice.

Nevertheless, clinical value of pharmacogenetic markers is not only limited to predict susceptibility to HDR in pre-treatment setting but may also be exploited as tests of exclusion, as has been proposed for the re-introduction of lumiracoxib, or differential diagnosis tools, as has been suggested for flucloxacillin-induced hepatitis [78]. Due 
Table 2 Main genetic variants discussed in this article.

\begin{tabular}{|c|c|c|c|c|c|c|c|}
\hline Gene $^{a}$ & Name $^{a}$ & Polymorphism ${ }^{a}$ & $\begin{array}{l}\text { Reference } \\
\text { rs }\end{array}$ & $\begin{array}{l}\text { Common name } \\
\text { of the allele }\end{array}$ & $\begin{array}{l}\text { Frequency of HLA alleles across } \\
\text { diverse populations [75] }\end{array}$ & $\begin{array}{l}\text { In vitro } \\
\text { function }\end{array}$ & $\begin{array}{l}\text { In vivo } \\
\text { function }\end{array}$ \\
\hline \multirow[t]{3}{*}{$\begin{array}{l}\text { Histocompatibility } \\
\text { complex (Chromosome } \\
\text { 6p21.3) }\end{array}$} & \multirow[t]{3}{*}{$\begin{array}{l}\text { Major } \\
\text { histocompatibility } \\
\text { complex, class I, A }\end{array}$} & *57:01 & N/A & HLA-B*57:01 & $\begin{array}{l}\text { West Europe: 5-10\%, } \\
\text { Mediterranean: } 1-4 \% \text {, UK: 5-10\%, } \\
\text { Middle East: 1-4\%, US Caucasian: } \\
5-10 \% \text {, US Asia: } 1-3 \% \text {, US } \\
\text { African-American: } 2-3 \% \text {, US } \\
\text { Hispanic: } 1-5 \% \text {, South American } \\
\text { Caucasian: } 5-10 \% \text {, Sub-Saharan } \\
\text { Africa: }<1 \% \text {, Australia: } 5-10 \% \text {, } \\
\text { Thailand: } 5-10 \% \text {, China: <1\%, } \\
\text { Japan: }<1 \% \text {, India: } 5-15 \%\end{array}$ & N/A & N/A \\
\hline & & *58:01 & $\mathrm{N} / \mathrm{A}$ & HLA-B*58:01 & $\begin{array}{l}\text { West Europe: } 1-4 \% \text {, } \\
\text { Mediterranean: } 1-4 \% \text {, UK: < } 1 \% \text {, } \\
\text { Middle East: } 5-10 \% \text {, US Caucasian: } \\
\text { 1-4\%, US Asia: } 5-10 \% \text {, US } \\
\text { African-American: } 5-10 \% \text {, US } \\
\text { Hispanic: } 1-4 \% \text {, South American } \\
\text { Caucasian: } 1-4 \% \text {, Sub-Saharan } \\
\text { Africa: } 5-15 \% \text {, Australia: } 1-4 \% \text {, } \\
\text { Thailand: } 5-15 \% \text {, China: 5-20\%, } \\
\text { Japan: < } 1 \% \text {, India: } 5-15 \%\end{array}$ & N/A & N/A \\
\hline & & *15:02 & N/A & HLA-B*15:02 & $\begin{array}{l}\text { West Europe: }<1 \% \text {, Mediterranean: } \\
<1 \% \text {, UK: }<1 \% \text {, Middle East: }<1 \% \text {, } \\
\text { US Caucasian: }<1 \% \text {, US Asia: } \\
5-10 \% \text {, US African-American: }<1 \% \text {, } \\
\text { US Hispanic: }<1 \% \text {, South American } \\
\text { Caucasian: }<1 \% \text {, Sub-Saharan } \\
\text { Africa: }<1 \% \text {, Australia: }<1 \% \text {, } \\
\text { Thailand: } 10-20 \% \text {, China: } 5-20 \% \text {, } \\
\text { Japan: }<1 \% \text {, India: } 5-15 \%\end{array}$ & N/A & N/A \\
\hline $\begin{array}{l}\text { From a practical point of v } \\
\text { "positive" or "negative", } \\
\text { risk of HDR. On the opposite, } \\
\text { [39]. N/A: not applicable. } \\
\text { a HGNC-approved gene nome }\end{array}$ & $\begin{array}{l}\text { HLA genotyping test } \\
\text { no intermediate phen } \\
\text { ositive'" result (hetero } \\
\text { ature (see http://wwv }\end{array}$ & $\begin{array}{l}\text { drug hypersensit } \\
\text { e. The absence } \\
\text { te and homozygc } \\
\text { nenames.org). }\end{array}$ & $\begin{array}{l}\text { should be } \\
\text { le tested all } \\
\text { ariants) me }\end{array}$ & $\begin{array}{l}\text { rpreted as follo } \\
\text { (reported as " } n \\
\text { that the drug is c }\end{array}$ & $\begin{array}{l}\text { ince HLA expression is co-dominant, } \\
\text { ive" on a genotyping test) indicates th } \\
\text { aindicated due to the increased risk of }\end{array}$ & $\begin{array}{l}\text { are repc } \\
\text { e patient } \\
\text { induced } h\end{array}$ & $\begin{array}{l}\text { as either } \\
\text { a very low } \\
\text { rsensitivity }\end{array}$ \\
\hline
\end{tabular}


to their pharmacological unpredictability and very low incidence, HDRs are usually recognized in the postmarketing phase of the drug life cycle leading to product withdrawal after important investment has been made in research and development. A systematic DNA banking during drug development phase could allow, if a HDR occurs, to retrospectively research a susceptibility genomic biomarker. In this context, successful identification of a predictive marker could avert withdrawal of the drug from the market by limiting the use of the drug to genetically tolerant subjects [79]. Last but not least, progresses in basic research on immunological mechanisms involved in HDRs could improve drug design, and lay the groundwork for the development of in vitro tests capable to identify harmful compounds in preclinical phases of drug development. These implementations would increase drug safety by predicting HDRs before their use in man, and, at the same time, would greatly reduce the cost of drug development [80].

\section{Disclosure of interest}

The authors declare that they have no competing interest.

\section{References}

[1] WHO World Health Organization. International drug monitoring. The role of hospital. Geneva, Switzerland: Technical report series; 1966. p. 425.

[2] Lazarou J, Pomeranz BH, Corey PN. Incidence of adverse drug reactions in hospitalized patients: a meta-analysis of prospective studies. JAMA 1998;279(15):1200-5.

[3] The European Commission document "Strengthening pharmacovigilance to reduce adverse effects of medicines". Brussels; 2008 [MEMO/08/782].

[4] Ernst FR, Grizzle AJ. Drug-related morbidity and mortality: updating the cost-of-illness model. J Am Pharm Assoc (Wash) 2001;41(2):192-9.

[5] Wysowski DK, Swartz L. Adverse drug event surveillance and drug withdrawals in the United States, 1969-2002: the importance of reporting suspected reactions. Arch Intern Med 2005;165(12):1363-9.

[6] Rawlins MD, Thompson JW. Pathogenesis of adverse drug reactions. In: Oxford University Press, editor. Textbook of adverse drug reactions. Oxford University Press ed; 1977.

[7] Johansson SG, Hourihane JO, Bousquet J, Bruijnzeel-Koomen C, Dreborg S, Haahtela T, et al. A revised nomenclature for allergy. An EAACI position statement from the EAACI nomenclature task force. Allergy 2001;56(9):813-24.

[8] Johansson SG, Bieber T, Dahl R, Friedmann PS, Lanier BQ, Lockey RF, et al. Revised nomenclature for allergy for global use: report of the nomenclature review Committee of the World Allergy Organization, October 2003. J Allergy Clin Immunol 2004;113(5):832-6.

[9] Coombs PRGP. Classification of allergic reactions responsible for clinical hypersensitivity and disease. Clinical aspects of immunology. Oxford, England: Oxford University Press; 1968. p. 575-96.

[10] Pichler WJ. Delayed drug hypersensitivity reactions. Ann Intern Med 2003;139(8):683-93.

[11] Pichler WJ, Adam J, Daubner B, Gentinetta T, Keller M, Yerly D. Drug hypersensitivity reactions: pathomechanism and clinical symptoms. Med Clin North Am 2010;94(4):645-64 [xv].

[12] Schnyder B, Mauri-Hellweg D, Zanni M, Bettens F, Pichler WJ. Direct, MHC-dependent presentation of the drug sulfamethoxazole to human alphabeta T cell clones. J Clin Invest 1997;100(1):136-41.

[13] Naisbitt DJ, Farrell J, Wong G, Depta JP, Dodd CC, Hopkins JE, et al. Characterization of drug-specific $T$ cells in lamotrigine hypersensitivity. J Allergy Clin Immunol 2003;111(6):1393-403.

[14] Zanni MP, von Greyerz S, Schnyder B, Brander KA, Frutig K, Hari $Y$, et al. HLA-restricted, processing- and metabolismindependent pathway of drug recognition by human alpha beta T lymphocytes. J Clin Invest 1998;102(8):1591-8.

[15] Pavlos R, Mallal S, Ostrov D, Pompeu Y, Phillips E. Fever, rash, and systemic symptoms: understanding the role of virus and HLA in severe cutaneous drug allergy. J Allergy Clin Immunol Pract 2014;2(1):21-33.

[16] Blackwell JM, Jamieson SE, Burgner D. HLA and infectious diseases. Clin Microbiol Rev 2009;22(2):370-85.

[17] Gough SC, Simmonds MJ. The HLA Region and autoimmune disease: associations and mechanisms of action. Curr Genomics 2007;8(7):453-65.

[18] Hetherington S, McGuirk S, Powell G, Cutrell A, Naderer O, Spreen B, et al. Hypersensitivity reactions during therapy with the nucleoside reverse transcriptase inhibitor abacavir. Clinical therapeutics 2001;23(10):1603-14.

[19] Escaut L, Liotier JY, Albengres E, Cheminot N, Vittecoq D. Abacavir rechallenge has to be avoided in case of hypersensitivity reaction. AIDS 1999;13(11):1419-20.

[20] Hetherington S, Hughes AR, Mosteller M, Shortino D, Baker KL, Spreen W, et al. Genetic variations in HLA-B region and hypersensitivity reactions to abacavir. Lancet 2002;359(9312):1121-2.

[21] Mallal S, Nolan D, Witt C, Masel G, Martin AM, Moore C, et al. Association between presence of HLA-B*5701, HLA-DR7, and HLA-DQ3 and hypersensitivity to HIV-1 reverse-transcriptase inhibitor abacavir. Lancet 2002;359(9308):727-32.

[22] Hughes DA, Vilar FJ, Ward CC, Alfirevic A, Park BK, Pirmohamed M. Cost-effectiveness analysis of HLA B*5701 genotyping in preventing abacavir hypersensitivity. Pharmacogenetics 2004;14(6):335-42.

[23] Rauch A, Nolan D, Martin A, McKinnon E, Almeida C, Mallal S. Prospective genetic screening decreases the incidence of abacavir hypersensitivity reactions in the Western Australian HIV cohort study. Clin Infec Dis 2006;43(1):99-102.

[24] Waters LJ, Mandalia S, Gazzard B, Nelson M. Prospective HLAB*5701 screening and abacavir hypersensitivity: a single centre experience. AIDS 2007;21(18):2533-4.

[25] Zucman D, Truchis P, Majerholc C, Stegman S, Caillat-Zucman S. Prospective screening for human leukocyte antigen-B*5701 avoids abacavir hypersensitivity reaction in the ethnically mixed French HIV population. J Acquir Immune Defic Syndr 2007;45(1):1-3.

[26] Mallal S, Phillips E, Carosi G, Molina JM, Workman C, Tomazic $J$, et al. HLA-B*5701 screening for hypersensitivity to abacavir. N Engl J Med 2008;358(6):568-79.

[27] Hung SI, Chung WH, Jee SH, Chen WC, Chang YT, Lee WR, et al. Genetic susceptibility to carbamazepine-induced cutaneous adverse drug reactions. Pharmacogenet Genomics 2006;16(4):297-306.

[28] Sousa-Pinto B, Pinto-Ramos J, Correia C, Goncalves-Costa G, Gomes L, Gil-Mata S, et al. Pharmacogenetics of abacavir hypersensitivity: a systematic review and meta-analysis of the association with HLA-B*57:01. J Allergy Clin Immunol 2015;136(4) [1092-4e3].

[29] Whirl-Carrillo M, McDonagh EM, Hebert JM, Gong L, Sangkuhl K, Thorn CF, et al. Pharmacogenomics knowledge for personalized medicine. Clin Pharmacol Ther 2012;92(4):414-7.

[30] Pavlos R, Mallal S, Ostrov D, Buus S, Metushi I, Peters B, et al. $T$ cell-mediated hypersensitivity reactions to drugs. Annu Rev Med 2015;66:439-54. 
[31] Yip VL, Marson AG, Jorgensen AL, Pirmohamed M, Alfirevic A. HLA genotype and carbamazepine-induced cutaneous adverse drug reactions: a systematic review. Clin Pharmacol Ther 2012;92(6):757-65.

[32] EMA. European Medicines Agency. http://www.ema.europa. eu/. [Accessed 18 November 2016].

[33] FDA. U.S. Food and Drug Administration. http://www.fda.gov/ [Accessed 18 November 2016].

[34] Daly AK, Donaldson PT, Bhatnagar P, Shen Y, Pe'er I, Floratos $A$, et al. HLA-B*5701 genotype is a major determinant of drug-induced liver injury due to flucloxacillin. Nat Genet 2009;41(7):816-9.

[35] Migueles SA, Sabbaghian MS, Shupert WL, Bettinotti MP, Marincola FM, Martino L, et al. HLA B*5701 is highly associated with restriction of virus replication in a subgroup of HIVinfected long term nonprogressors. Proc Natl Acad Sci U S A 2000;97(6):2709-14.

[36] Fellay J, Shianna KV, Ge D, Colombo S, Ledergerber B, Weale M, et al. A whole-genome association study of major determinants for host control of HIV-1. Science 2007;317(5840):944-7.

[37] UK Collaborative HIV Cohort Study Steering Committee. HLA $B * 5701$ status, disease progression, and response to antiretroviral therapy. AIDS 2013;27(16):2587-92.

[38] Mockenhaupt M. The current understanding of Stevens-Johnson syndrome and toxic epidermal necrolysis. Expert Rev Clin Immunol 2011;7(6):803-13 [quiz 14-5].

[39] Downey A, Jackson C, Harun N, Cooper A. Toxic epidermal necrolysis: review of pathogenesis and management. J Am Acad Dermatol 2012;66(6):995-1003.

[40] Dao RL, Su SC, Chung WH. Recent advances of pharmacogenomics in severe cutaneous adverse reactions: immune and nonimmune mechanisms. Asia Pac Allergy 2015;5(2):59-67.

[41] Harr T, French LE. Toxic epidermal necrolysis and StevensJohnson syndrome. Orphanet J Rare Dis 2010;5:39.

[42] Barbarino JM, Kroetz DL, Klein TE, Altman RB. PharmGKB summary: very important pharmacogene information for human leukocyte antigen B. Pharmacogenet genomics 2015;25(4):205-21.

[43] Chung WH, Hung SI, Hong HS, Hsih MS, Yang LC, Ho HC, et al. Medical genetics: a marker for Stevens-Johnson syndrome. Nature 2004;428(6982):486.

[44] Chung WH, Hung SI. Recent advances in the genetics and immunology of Stevens-Johnson syndrome and toxic epidermal necrosis. J Dermatol Sci 2012;66(3):190-6.

[45] Cheng CY, Su SC, Chen CH, Chen WL, Deng ST, Chung WH. HLA associations and clinical implications in T-cell mediated drug hypersensitivity reactions: an updated review. J Immunol Res 2014;2014:565320.

[46] Man CB, Kwan P, Baum L, Yu E, Lau KM, Cheng AS, et al. Association between HLA-B*1502 allele and antiepileptic drug-induced cutaneous reactions in Han Chinese. Epilepsia 2007;48(5):1015-8.

[47] Wu XT, Hu FY, An DM, Yan B, Jiang X, Kwan P, et al. Association between carbamazepine-induced cutaneous adverse drug reactions and the HLA-B*1502 allele among patients in central China. Epilepsy Behav 2010;19(3):405-8.

[48] Wang Q, Zhou JQ, Zhou LM, Chen ZY, Fang ZY, Chen SD, et al. Association between HLA-B*1502 allele and carbamazepineinduced severe cutaneous adverse reactions in Han people of southern China mainland. Seizure 2011;20(6):446-8.

[49] Tassaneeyakul W, Tiamkao S, Jantararoungtong T, Chen P, Lin SY, Chen WH, et al. Association between HLA-B*1502 and carbamazepine-induced severe cutaneous adverse drug reactions in a Thai population. Epilepsia 2010;51(5):926-30.

[50] Locharernkul C, Loplumlert J, Limotai C, Korkij W, Desudchit T, Tongkobpetch S, et al. Carbamazepine and phenytoin induced Stevens-Johnson syndrome is associated with HLA-B*1502 allele in Thai population. Epilepsia 2008;49(12):2087-91.
[51] Then SM, Rani ZZ, Raymond AA, Ratnaningrum S, Jamal R. Frequency of the HLA-B*1502 allele contributing to carbamazepine-induced hypersensitivity reactions in a cohort of Malaysian epilepsy patients. Asian Pac J Allergy Immunol 2011;29(3):290-3.

[52] Mehta TY, Prajapati LM, Mittal B, Joshi CG, Sheth JJ, Patel DB, et al. Association of HLA-B*1502 allele and carbamazepineinduced Stevens-Johnson syndrome among Indians. Indian J Dermatol Venereol Leprol 2009;75(6):579-82.

[53] Kulkantrakorn K, Tassaneeyakul W, Tiamkao S, Jantararoungtong T, Prabmechai N, Vannaprasaht S, et al. HLA-B*1502 strongly predicts carbamazepine-induced Stevens-Johnson syndrome and toxic epidermal necrolysis in Thai patients with neuropathic pain. Pain Pract 2012;12(3):202-8.

[54] Grover S, Kukreti R. HLA alleles and hypersensitivity to carbamazepine: an updated systematic review with meta-analysis. Pharmacogenet Genomics 2014;24(2):94-112.

[55] Tangamornsuksan W, Chaiyakunapruk N, Somkrua R, Lohitnavy M, Tassaneeyakul W. Relationship between the HLA-B*1502 allele and carbamazepine-induced Stevens-Johnson syndrome and toxic epidermal necrolysis: a systematic review and metaanalysis. JAMA Dermatol 2013;149(9):1025-32.

[56] Bloch KM, Sills GJ, Pirmohamed M, Alfirevic A. Pharmacogenetics of antiepileptic drug-induced hypersensitivity. Pharmacogenomics 2014;15(6):857-68.

[57] Chen P, Lin JJ, Lu CS, Ong CT, Hsieh PF, Yang CC, et al. Carbamazepine-induced toxic effects and HLA-B*1502 screening in Taiwan. N Engl J Med 2011;364(12):1126-33.

[58] Ozeki T, Mushiroda T, Yowang A, Takahashi A, Kubo M, Shirakata Y, et al. Genome-wide association study identifies HLA-A*3101 allele as a genetic risk factor for carbamazepine-induced cutaneous adverse drug reactions in Japanese population. Hum Mol Genet 2011;20(5):1034-41.

[59] McCormack M, Alfirevic A, Bourgeois S, Farrell JJ, Kasperaviciute D, Carrington M, et al. HLA-A*3101 and carbamazepineinduced hypersensitivity reactions in Europeans. N Engl J Med 2011;364(12):1134-43.

[60] Alfirevic A, Jorgensen AL, Williamson PR, Chadwick DW, Park BK, Pirmohamed M. HLA-B locus in Caucasian patients with carbamazepine hypersensitivity. Pharmacogenomics 2006;7(6):813-8.

[61] Niihara H, Kakamu T, Fujita Y, Kaneko S, Morita E. HLAA31 strongly associates with carbamazepine-induced adverse drug reactions but not with carbamazepine-induced lymphocyte proliferation in a Japanese population. J Dermatol 2012;39(7):594-601.

[62] Hung SI, Chung WH, Liu ZS, Chen CH, Hsih MS, Hui RC, et al. Common risk allele in aromatic antiepileptic-drug induced Stevens-Johnson syndrome and toxic epidermal necrolysis in Han Chinese. Pharmacogenomics 2010;11(3):349-56.

[63] Liao WP, Shi YW, Min FL. HLA-B*1502 screening and toxic effects of carbamazepine. N Engl J Med 2011;365(7):672-3 [author reply 3].

[64] Hershfield MS, Callaghan JT, Tassaneeyakul W, Mushiroda T, Thorn CF, Klein TE, et al. Clinical pharmacogenetics implementation consortium guidelines for human leukocyte antigen-B genotype and allopurinol dosing. Clin Pharmacol Ther 2013;93(2):153-8.

[65] Lee MT, Mahasirimongkol S, Zhang Y, Suwankesawong W, Chaikledkaew U, Pavlidis C, et al. Clinical application of pharmacogenomics: the example of HLA-based drug-induced toxicity. Public Health Genomics 2014;17(5-6):248-55.

[66] Hung SI, Chung WH, Liou LB, Chu CC, Lin M, Huang HP, et al. HLA-B*5801 allele as a genetic marker for severe cutaneous adverse reactions caused by allopurinol. Proc Natl Acad Sci U S A 2005;102(11):4134-9.

[67] Tohkin M, Kaniwa N, Saito Y, Sugiyama E, Kurose K, Nishikawa J, et al. A whole-genome association study of major 
determinants for allopurinol-related Stevens-Johnson syndrome and toxic epidermal necrolysis in Japanese patients. Pharmacogenomics J 2013;13(1):60-9.

[68] Tassaneeyakul W, Jantararoungtong T, Chen P, Lin PY, Tiamkao $S$, Khunarkornsiri $U$, et al. Strong association between HLAB*5801 and allopurinol-induced Stevens-Johnson syndrome and toxic epidermal necrolysis in a Thai population. Pharmacogenet Genomics 2009;19(9):704-9.

[69] Cao ZH, Wei ZY, Zhu QY, Zhang JY, Yang L, Qin SY, et al. HLA$B * 58: 01$ allele is associated with augmented risk for both mild and severe cutaneous adverse reactions induced by allopurinol in Han Chinese. Pharmacogenomics 2012;13(10):1193-201.

[70] Kaniwa N, Saito Y, Aihara M, Matsunaga K, Tohkin M, Kurose $\mathrm{K}$, et al. HLA-B locus in Japanese patients with anti-epileptics and allopurinol-related Stevens-Johnson syndrome and toxic epidermal necrolysis. Pharmacogenomics 2008;9(11):1617-22.

[71] Kang HR, Jee YK, Kim YS, Lee CH, Jung JW, Kim SH, et al. Positive and negative associations of HLA class I alleles with allopurinol-induced SCARs in Koreans. Pharmacogenet Genomics 2011;21(5):303-7.

[72] Lonjou C, Borot N, Sekula P, Ledger N, Thomas L, Halevy S, et al. A European study of HLA-B in Stevens-Johnson syndrome and toxic epidermal necrolysis related to five high-risk drugs. Pharmacogenet Genomics 2008;18(2):99-107.

[73] Somkrua R, Eickman EE, Saokaew S, Lohitnavy M, Chaiyakunapruk N. Association of HLA-B*5801 allele and allopurinol-induced Stevens Johnson syndrome and toxic epidermal necrolysis: a systematic review and meta-analysis. BMC Med Genet 2011;12:118.

[74] Khanna D, Fitzgerald JD, Khanna PP, Bae S, Singh MK, Neogi T, et al. 2012 American College of Rheumatology guidelines for management of gout. Part 1: systematic nonpharmacologic and pharmacologic therapeutic approaches to hyperuricemia. Arthritis Care Res (Hoboken) 2012;64(10):1431-46.

[75] Gonzalez-Galarza FF, Takeshita LY, Santos EJ, Kempson F, Maia MH, da Silva AL, et al. Allele frequency net 2015 update: new features for HLA epitopes, KIR and disease and HLA adverse drug reaction associations. Nucleic Acids Res 2015;43:D784-8 [Database issue].

[76] Phillips EJ, Mallal SA. Pharmacogenetics of drug hypersensitivity. Pharmacogenomics 2010;11(7):973-87.

[77] Karlin E, Phillips E. Genotyping for severe drug hypersensitivity. Curr Allergy Asthma Rep 2014;14(3):418.

[78] Alfirevic A, Pirmohamed M. Drug induced hypersensitivity and the HLA complex. Pharmaceuticals 2011;4(1):69-90.

[79] Becquemont L. HLA: a pharmacogenomics success story. Pharmacogenomics 2010;11(3):277-81.

[80] White KD, Chung WH, Hung SI, Mallal S, Phillips EJ. Evolving models of the immunopathogenesis of $\mathrm{T}$ cell-mediated drug allergy: the role of host, pathogens, and drug response. J Allergy Clin Immunol 2015;136(2):219-34 [quiz 35]. 